Supporting Information

\title{
Bioaccumulation and Trophic Transfer of Polybrominated Diphenyl Ethers and Their Hydroxylated and Methoxylated Analogues in Polar Marine Food Webs
}

Huizhong Sun ${ }^{\mathrm{a}, \mathrm{b}}$, Yingming $\mathrm{Li}^{\mathrm{b}, \mathrm{c}, *}$, Yanfen Hao ${ }^{\mathrm{b}, \mathrm{c}}$, Ying Zhu ${ }^{\mathrm{b}}$, Ruiqiang Yang ${ }^{\mathrm{b}}$, Pu Wang ${ }^{\mathrm{d}}$, Qinghua Zhang ${ }^{\mathrm{b}, \mathrm{c}, \mathrm{e}}$, Guibin Jiang ${ }^{\mathrm{b}, \mathrm{c}}$

${ }^{a}$ National Research Center for Geoanalysis, Beijing 100037, China

${ }^{b}$ State Key Laboratory of Environmental Chemistry and Ecotoxicology, Research Center for Eco-Environmental Sciences, Chinese Academy of Sciences, Beijing 100085, China

'University of Chinese Academy of Sciences, Beijing 100049, China

${ }^{d}$ Hubei Key Laboratory of Environmental and Health Effects of Persistent Toxic Substances, Institute of Environment and Health, Jianghan University, Wuhan 430056, China ${ }^{e}$ Institute of Environment and Health, Hangzhou Institute for Advanced Study, University of Chinese Academy of Sciences, Hangzhou 310000, China

Number of Pages: 11

Number of Tables: 6

Number of Figures: 4 

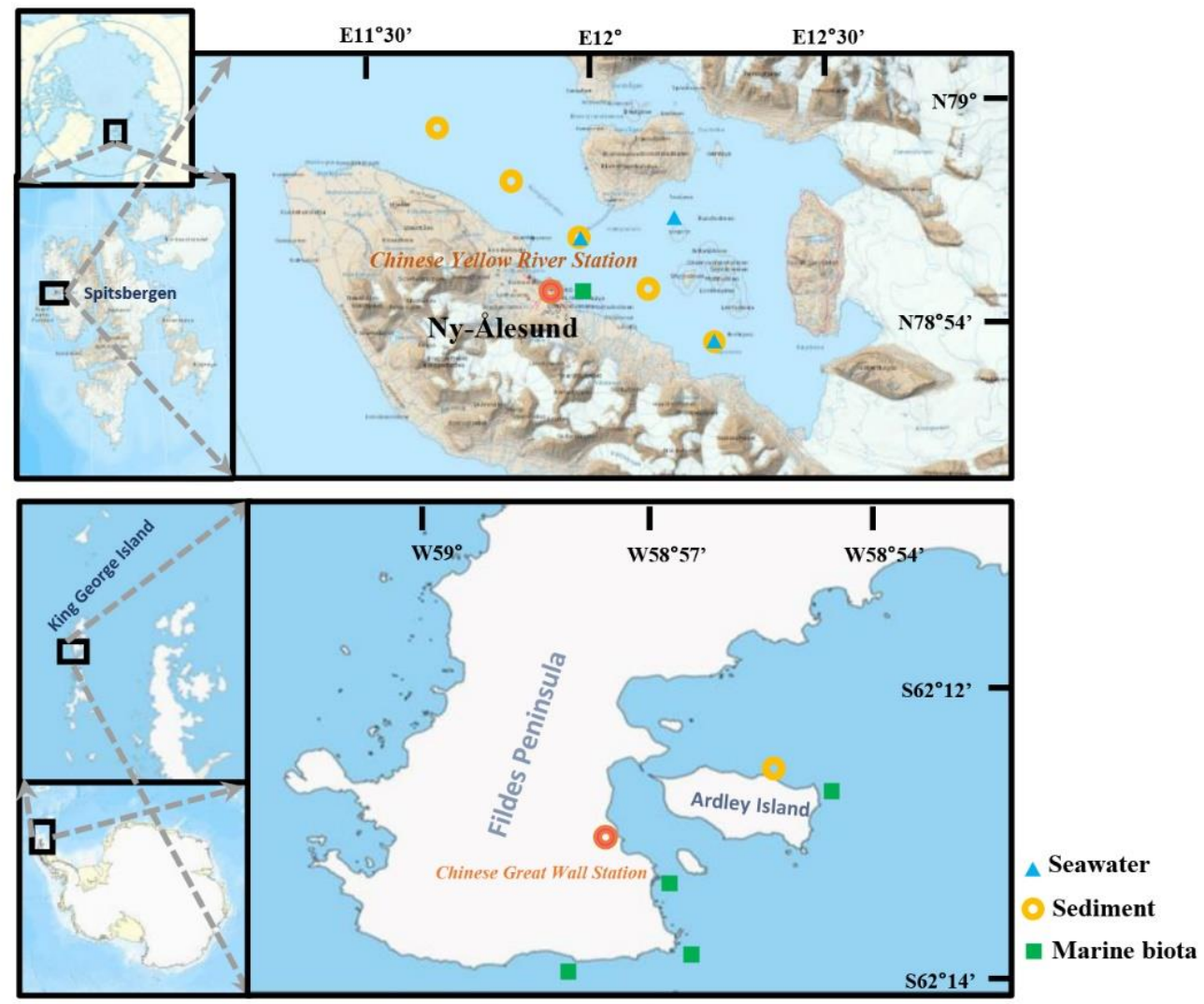

Figure S1. Map of study area in the Arctic and Antarctic regions. 
Table S1. Retention time (RT), MRM transitions and collision energy (CE) of PBDE congeners and ${ }^{13} \mathrm{C}$-internal standards

\begin{tabular}{|c|c|c|c|c|c|c|c|}
\hline \multirow{2}{*}{ No. } & \multirow{2}{*}{ Compound } & \multirow{2}{*}{$\begin{array}{c}\mathrm{RT} \\
(\mathrm{min})\end{array}$} & \multicolumn{2}{|c|}{ Quantitative analysis } & \multicolumn{2}{|c|}{ Qualitative analysis } & \multirow{2}{*}{$\begin{array}{c}\text { Quant/Qual } \\
\text { Ratio (\%) }\end{array}$} \\
\hline & & & MRM & $\mathrm{CE}(\mathrm{V})$ & MRM & $\mathrm{CE}(\mathrm{V})$ & \\
\hline 1 & ${ }^{13} \mathrm{C}_{12}$-BDE-28 & 11.95 & $417.8>257.9$ & 20 & $419.8>259.9$ & 20 & 77 \\
\hline 2 & BDE-28 & 11.95 & $405.8>245.9$ & 20 & $407.8>247.9$ & 20 & 79 \\
\hline 3 & ${ }^{13} \mathrm{C}_{12}-\mathrm{PCB}-138$ & 12.57 & $371.9>301.9$ & 24 & $369.9>299.9$ & 24 & 81 \\
\hline 4 & ${ }^{13} \mathrm{C}_{12}$-BDE-47 & 13.92 & $497.7>337.7$ & 24 & $499.7>339.7$ & 24 & 42 \\
\hline 5 & BDE-47 & 13.92 & $485.7>325.7$ & 24 & $487.7>327.7$ & 24 & 46 \\
\hline 6 & ${ }^{13} \mathrm{C}_{12}-\mathrm{BDE}-100$ & 15.73 & $575.6>415.7$ & 24 & $577.6>417.7$ & 24 & 81 \\
\hline 7 & BDE-100 & 15.73 & $563.6>403.7$ & 24 & $565.6>405.7$ & 24 & 84 \\
\hline 8 & ${ }^{13} \mathrm{C}_{12}-\mathrm{PCB}-194$ & 15.87 & $439.8>369.8$ & 24 & $441.8>371.8$ & 24 & 80 \\
\hline 9 & ${ }^{13} \mathrm{C}_{12}$-BDE-99 & 16.38 & $575.6>415.7$ & 26 & $577.6>417.7$ & 26 & 85 \\
\hline 10 & BDE-99 & 16.38 & $563.6>403.7$ & 26 & $565.6>405.7$ & 26 & 87 \\
\hline 11 & ${ }^{13} \mathrm{C}_{12}-\mathrm{BDE}-154$ & 18.09 & $655.6>495.7$ & 26 & $657.6>497.7$ & 26 & 61 \\
\hline 12 & BDE-154 & 18.09 & $643.6>483.7$ & 26 & $645.6>485.7$ & 26 & 64 \\
\hline 13 & ${ }^{13} \mathrm{C}_{12}$-BDE-153 & 19.10 & $655.6>495.7$ & 26 & $657.6>497.7$ & 26 & 59 \\
\hline 14 & BDE-153 & 19.11 & $643.6>483.7$ & 26 & $645.6>485.7$ & 26 & 55 \\
\hline 15 & ${ }^{13} \mathrm{C}_{12}-\mathrm{BDE}-183$ & 21.45 & $733.4>573.6$ & 30 & $735.4>575.6$ & 30 & 87 \\
\hline 16 & BDE-183 & 21.46 & $721.4>561.6$ & 30 & $723.4>563.6$ & 30 & 90 \\
\hline
\end{tabular}


Table S2. Retention time (RT), MRM transitions and collision energy (CE) of MeO-PBDE congeners and internal standards

\begin{tabular}{|c|c|c|c|c|c|c|c|}
\hline \multirow{2}{*}{ No. } & \multirow{2}{*}{ Compound } & \multirow{2}{*}{$\begin{array}{c}\mathrm{RT} \\
(\mathrm{min})\end{array}$} & \multicolumn{2}{|c|}{ Quantitative analysis } & \multicolumn{2}{|c|}{ Qualitative analysis } & \multirow{2}{*}{$\begin{array}{c}\text { Quant/Qua } \\
\text { Ratio (\%) }\end{array}$} \\
\hline & & & MRM & $\mathrm{CE}(\mathrm{V})$ & MRM & $\mathrm{CE}(\mathrm{V})$ & \\
\hline 1 & 2'-MeO-BDE-28 & 17.02 & $436.0>276.0$ & 15 & $438.0>278.0$ & 15 & 84 \\
\hline 2 & 3'-MeO-BDE-28 & 17.53 & $436.0>276.0$ & 15 & $438.0>278.0$ & 15 & 81 \\
\hline 3 & 2'-MeO-BDE-68 & 18.78 & $515.5>355.5$ & 15 & $513.5>353.5$ & 15 & 54 \\
\hline 4 & ${ }^{13} \mathrm{C}_{12}-6-\mathrm{MeO}-\mathrm{BDE}-47$ & 19.14 & $527.5>367.5$ & 15 & $525.5>365.5$ & 15 & 62 \\
\hline 5 & 6-MeO-BDE-47 & 19.14 & $515.5>355.5$ & 15 & $513.5>353.5$ & 15 & 58 \\
\hline 6 & 3-MeO-BDE-47 & 19.64 & $515.5>355.5$ & 15 & $513.5>353.5$ & 15 & 64 \\
\hline 7 & 5-MeO-BDE-47 & 19.81 & $515.5>355.5$ & 15 & $513.5>353.5$ & 15 & 58 \\
\hline 8 & 4'-MeO-BDE-49 & 19.95 & $515.5>355.5$ & 15 & $513.5>353.5$ & 15 & 56 \\
\hline 9 & ${ }^{13} \mathrm{C}_{12}-6^{\prime}-\mathrm{MeO}-\mathrm{BDE}-100$ & 20.82 & $605.5>445.5$ & 15 & $607.5>447.5$ & 15 & 96 \\
\hline 10 & 4-MeO-BDE-42 & 21.08 & $515.5>515.5$ & 15 & $513.5>353.5$ & 15 & 23 \\
\hline 11 & 5'-MeO-BDE-100 & 21.34 & $593.5>433.5$ & 15 & $595.5>435.5$ & 15 & 91 \\
\hline 12 & 4'-MeO-BDE-103 & 21.53 & $595.5>435.5$ & 15 & $593.5>433.5$ & 15 & 114 \\
\hline 13 & 6'-MeO-BDE-99 & 21.62 & $595.5>435.5$ & 15 & $593.5>433.5$ & 15 & 111 \\
\hline 14 & 5'-MeO-BDE-99 & 22.50 & $593.5>433.5$ & 15 & $595.5>435.5$ & 15 & 87 \\
\hline 15 & 4'-MeO-BDE-101 & 22.67 & $595.5>435.5$ & 15 & $593.5>433.5$ & 15 & 107 \\
\hline 16 & 6-MeO-BDE-85 & 23.01 & $593.5>433.5$ & 15 & $595.5>435.5$ & 15 & 88 \\
\hline 17 & BDE-128 & 25.77 & $643.0>483.7$ & 15 & $641.0>481.7$ & 15 & 78 \\
\hline
\end{tabular}


Table S3. Details on samples: total organic carbon (TOC) content (\%), lipid content (\% of dry weight) and stable isotopic compositions.

\begin{tabular}{|c|c|c|c|c|c|c|}
\hline Location & Matrice & Sampling date & TOC $(\%)$ & Lipid content $(\%)$ & $\delta^{13} \mathrm{C}(\%)$ & $\delta^{15} \mathrm{~N}(\%)$ \\
\hline \multirow{7}{*}{ Arctic } & seaweed (Fucus distichus) & July 2012 & & $1.0 \pm 0.9$ & $-16.4 \pm 1.9$ & $4.4 \pm 0.4$ \\
\hline & amphipod (Themisto libellula) & July 2012 & & $6.2 \pm 0.3$ & $-19.7 \pm 0.2$ & $7.4 \pm 0.1$ \\
\hline & barnacle (Balanus Balanus) & July 2012 & & $1.0 \pm 0.2$ & $-17.7 \pm 3.4$ & $8.4 \pm 0.6$ \\
\hline & jellyfish (Cyanea sp.) & July 2012 & & $4.7 \pm 2.1$ & $-23.3 \pm 0.5$ & $9.4 \pm 0.4$ \\
\hline & Atlantic cod (Gadus morhua) & July 2012 & & $3.6 \pm 0.5$ & $-20.6 \pm 0.2$ & $12.7 \pm 0.4$ \\
\hline & seawater & July 2012 & & & & \\
\hline & sediment & July 2012 & $0.77 \pm 0.40$ & & & \\
\hline \multirow{5}{*}{ Antarctic } & algae (Palmaria decipiens) & January 2014 & & $0.90 \pm 0.46$ & $-21.0 \pm 6.4$ & $3.9 \pm 0.7$ \\
\hline & limpet (Nacella concinna) & January 2014 & & $11.7 \pm 4.5$ & $-17.1 \pm 1.4$ & $6.5 \pm 0.8$ \\
\hline & starfish (Diplasterias brucei) & January 2014 & & $11.5 \pm 3.7$ & $-16.6 \pm 2.4$ & $8.5 \pm 0.6$ \\
\hline & Patagonian toothfish (Dissostichus eleginoides) & January 2014 & & $5.5 \pm 2.4$ & $-20.6 \pm 1.2$ & $10.8 \pm 0.5$ \\
\hline & sediment & January 2010 & 3.3 & & & \\
\hline
\end{tabular}


Table S4. Detection frequency of PBDEs and metabolites in the Arctic and Antarctic marine matrices.

\begin{tabular}{|c|c|c|c|c|c|c|}
\hline \multirow{2}{*}{ No. } & \multirow{2}{*}{ Compound } & \multicolumn{3}{|c|}{ Arctic } & \multicolumn{2}{|c|}{ Antarctic } \\
\hline & & biota & sediment & seawater & biota & sediment \\
\hline 1 & BDE-28 & $20 / 20$ & $5 / 5$ & $3 / 3$ & $30 / 31$ & $1 / 1$ \\
\hline 2 & BDE-47 & $20 / 20$ & $5 / 5$ & $3 / 3$ & $31 / 31$ & $1 / 1$ \\
\hline 3 & BDE-99 & $20 / 20$ & $5 / 5$ & $3 / 3$ & $31 / 31$ & $1 / 1$ \\
\hline 4 & BDE-100 & $20 / 20$ & $5 / 5$ & $2 / 3$ & $26 / 31$ & $1 / 1$ \\
\hline 5 & BDE-153 & $20 / 20$ & n.d. & n.d. & $31 / 31$ & n.d. \\
\hline 6 & BDE-154 & $19 / 20$ & n.d. & n.d. & $28 / 31$ & n.d. \\
\hline 7 & BDE-183 & $15 / 20$ & n.d. & $1 / 3$ & $28 / 31$ & n.d. \\
\hline 1 & 2'-MeO-BDE-28 & $1 / 20$ & $4 / 5$ & n.d. & $14 / 31$ & $1 / 1$ \\
\hline 2 & 3'-MeO-BDE-28 & $4 / 20$ & n.d. & n.d. & n.d. & n.d. \\
\hline 3 & 2'-MeO-BDE-68 & $15 / 20$ & $5 / 5$ & n.d. & $25 / 31$ & $1 / 1$ \\
\hline 4 & 6-MeO-BDE-47 & $19 / 20$ & $5 / 5$ & n.d. & $30 / 31$ & $1 / 1$ \\
\hline 5 & 3-MeO-BDE-47 & $1 / 20$ & $2 / 5$ & n.d. & $1 / 31$ & $1 / 1$ \\
\hline 6 & 5-MeO-BDE-47 & $1 / 20$ & n.d. & n.d. & $1 / 31$ & n.d. \\
\hline 7 & 4'-MeO-BDE-49 & $1 / 20$ & n.d. & n.d. & $4 / 31$ & n.d. \\
\hline 8 & 4-MeO-BDE-42 & n.d. & n.d. & n.d. & n.d. & n.d. \\
\hline 9 & 5'-MeO-BDE-100 & n.d. & n.d. & n.d. & n.d. & n.d. \\
\hline 10 & 4'-MeO-BDE-103 & n.d. & $1 / 5$ & n.d. & $3 / 31$ & $1 / 1$ \\
\hline 11 & 6'-MeO-BDE-99 & n.d. & n.d. & n.d. & n.d. & n.d. \\
\hline 12 & 5'-MeO-BDE-99 & n.d. & n.d. & n.d. & n.d. & n.d. \\
\hline 13 & 4'-MeO-BDE-101 & n.d. & n.d. & n.d. & n.d. & n.d. \\
\hline 14 & 6-MeO-BDE-85 & n.d. & n.d. & n.d. & $2 / 31$ & $1 / 1$ \\
\hline 1 & 2'-OH-BDE-28 & $5 / 20$ & $5 / 5$ & n.d. & $20 / 31$ & $1 / 1$ \\
\hline 2 & 3'-OH-BDE-28 & $1 / 20$ & n.d. & n.d. & $15 / 31$ & n.d. \\
\hline 3 & 2'-OH-BDE-68 & $19 / 20$ & $5 / 5$ & $3 / 3$ & $30 / 31$ & $1 / 1$ \\
\hline 4 & 6-OH-BDE-47 & $20 / 20$ & $5 / 5$ & $3 / 3$ & $30 / 31$ & $1 / 1$ \\
\hline 5 & 3-OH-BDE-47 & $7 / 20$ & $4 / 5$ & n.d. & $20 / 31$ & $1 / 1$ \\
\hline 6 & 5-OH-BDE-47 & $6 / 20$ & $2 / 5$ & n.d. & $10 / 31$ & n.d. \\
\hline 7 & 4'-OH-BDE-49 & $9 / 20$ & n.d. & n.d. & $12 / 31$ & n.d. \\
\hline 8 & 4-OH-BDE-42 & n.d. & n.d. & n.d. & n.d. & n.d. \\
\hline 9 & 5'-OH-BDE-100 & n.d. & n.d. & n.d. & n.d. & n.d. \\
\hline 10 & 4'-OH-BDE-103 & n.d. & $2 / 5$ & n.d. & $16 / 31$ & $1 / 1$ \\
\hline 11 & 6'-OH-BDE-99 & n.d. & n.d. & n.d. & n.d. & n.d. \\
\hline 12 & 5'-OH-BDE-99 & n.d. & n.d. & n.d. & n.d. & n.d. \\
\hline 13 & 4'-OH-BDE-101 & n.d. & n.d. & n.d. & n.d. & n.d. \\
\hline 14 & 6-OH-BDE-85 & n.d. & $5 / 5$ & n.d. & $15 / 31$ & $1 / 1$ \\
\hline
\end{tabular}


Table S5. $\log$ BAF (L/kg lipid) and BSAF (g TOC/g lipid) values (arithmetic mean and standard deviation) of each congener of PBDEs and metabolites in the Arctic organisms.

\begin{tabular}{|c|c|c|c|c|c|c|c|c|c|c|c|}
\hline & \multirow{2}{*}{ Compound } & \multicolumn{2}{|c|}{ Seaweed } & \multicolumn{2}{|c|}{ Amphipod } & \multicolumn{2}{|c|}{ Barnacle } & \multicolumn{2}{|c|}{ Jellyfish } & \multicolumn{2}{|c|}{ Cod } \\
\hline & & Mean & $S D$ & Mean & $S D$ & Mean & $S D$ & Mean & $S D$ & Mean & $S D$ \\
\hline \multirow{8}{*}{$\begin{array}{l}\log _{10} \\
\text { BAF }\end{array}$} & BDE-28 & 5.5 & 0.2 & 5.5 & 0.1 & 5.8 & 0.2 & 5.1 & 0.4 & 5.6 & 0.2 \\
\hline & BDE-47 & 6.1 & 0.4 & 6.5 & 0.1 & 6.7 & 0.2 & 5.4 & 0.4 & 6.0 & 0.2 \\
\hline & BDE-99 & 5.8 & 0.5 & 6.1 & 0.1 & 6.3 & 0.2 & 4.8 & 0.3 & 4.8 & 0.4 \\
\hline & BDE-100 & 6.5 & 0.5 & 7.0 & 0.1 & 7.1 & 0.2 & 5.5 & 0.2 & 6.2 & 0.2 \\
\hline & $\sum$ PBDEs & 5.9 & 0.5 & 6.3 & 0.1 & 6.4 & 0.2 & 5.2 & 0.4 & 5.7 & 0.2 \\
\hline & 2'-OH-BDE-68 & 4.8 & 0.5 & 5.1 & 0.1 & 5.3 & 0.4 & 4.8 & 0.4 & 4.5 & 0.2 \\
\hline & 6-OH-BDE-47 & 5.4 & 0.5 & 5.8 & 0.1 & 5.8 & 0.4 & 5.8 & 0.2 & 5.2 & 0.2 \\
\hline & $\sum \mathrm{OH}-\mathrm{PBDEs}$ & 5.6 & 0.3 & 5.9 & 0.1 & 6.2 & 0.2 & 5.8 & 0.2 & 5.3 & 0.3 \\
\hline \multirow{11}{*}{ BSAF } & BDE-28 & 1.5 & 0.6 & 1.3 & 0.2 & 2.9 & 1.3 & 0.82 & 0.80 & 1.7 & 0.5 \\
\hline & BDE-47 & 3.1 & 2.9 & 5.6 & 0.3 & 8.4 & 2.4 & 0.59 & 0.48 & 2.0 & 0.5 \\
\hline & BDE-99 & 32 & 14 & 87 & 8 & 166 & 58 & 5.53 & 2.91 & 5.6 & 4.1 \\
\hline & BDE-100 & 2.3 & 3.3 & 4.7 & 0.3 & 6.2 & 1.9 & 0.14 & 0.03 & 0.7 & 0.2 \\
\hline & $\sum$ PBDEs & 5.5 & 6.7 & 7.6 & 0.4 & 12.2 & 3.6 & 0.98 & 0.95 & 1.9 & 0.5 \\
\hline & 2'-MeO-BDE-68 & I & & 0.20 & 0.03 & 0.15 & 0.06 & 0.074 & 0.005 & 0.23 & 0.09 \\
\hline & 6-MeO-BDE-47 & 0.014 & 0.012 & 0.10 & 0.01 & 0.09 & 0.03 & 0.034 & 0.007 & 0.14 & 0.06 \\
\hline & $\sum \mathrm{MeO}-\mathrm{PBDEs}$ & 0.013 & 0.013 & 0.12 & 0.01 & 0.09 & 0.03 & 0.037 & 0.006 & 0.14 & 0.05 \\
\hline & 2'-OH-BDE-68 & 0.026 & 0.018 & 0.037 & 0.008 & 0.071 & 0.063 & 0.018 & 0.010 & 0.007 & 0.005 \\
\hline & 6-OH-BDE-47 & 0.012 & 0.010 & 0.023 & 0.004 & 0.026 & 0.015 & 0.020 & 0.008 & 0.005 & 0.002 \\
\hline & $\sum \mathrm{OH}-\mathrm{PBDEs}$ & 0.013 & 0.008 & 0.023 & 0.003 & 0.039 & 0.010 & 0.018 & 0.007 & 0.005 & 0.002 \\
\hline
\end{tabular}


Table S6. BSAF (g TOC/g lipid) values (arithmetic mean and standard deviation) of each congener of PBDEs and metabolites in the Antarctic organisms.

\begin{tabular}{cccccccccc}
\hline \multirow{2}{*}{ Compound } & \multicolumn{2}{c}{ Algae } & \multicolumn{2}{c}{ Limpet } & \multicolumn{2}{c}{ Starfish } & \multicolumn{2}{c}{ Toothfish } \\
\cline { 2 - 9 } & Mean & $S D$ & Mean & $S D$ & Mean & $S D$ & Mean & $S D$ \\
\hline BDE-28 & 2.2 & 3.1 & 0.26 & 0.27 & 0.35 & 0.30 & 0.81 & 1.23 \\
BDE-47 & 5.1 & 6.7 & 0.59 & 0.47 & 0.88 & 1.07 & 1.9 & 1.3 \\
BDE-99 & 1.3 & 1.4 & 0.18 & 0.20 & 0.39 & 0.64 & 0.53 & 0.48 \\
BDE-100 & 3.8 & 4.4 & 0.47 & 0.78 & 6.4 & 6.0 & 8.1 & 7.3 \\
LPBDEs & 3.8 & 4.2 & 2.6 & 3.5 & 0.85 & 0.83 & 12 & 25 \\
BSAF & 2'-MeO-BDE-68 & 0.020 & 0.026 & 0.07 & 0.09 & 0.003 & 0.004 & 0.019 & 0.016 \\
& 6-MeO-BDE-47 & 0.005 & 0.006 & 0.010 & 0.003 & 0.006 & 0.005 & 0.043 & 0.027 \\
LMeO-PBDEs & 0.002 & 0.002 & 0.004 & 0.002 & 0.002 & 0.001 & 0.013 & 0.008 \\
2'-OH-BDE-68 & 0.047 & 0.067 & 0.26 & 0.19 & 0.10 & 0.04 & 0.0015 & 0.0004 \\
6-OH-BDE-47 & 0.035 & 0.039 & 0.46 & 0.25 & 0.12 & 0.05 & 0.014 & 0.010 \\
LOH-PBDEs & 0.020 & 0.023 & 0.18 & 0.10 & 0.053 & 0.020 & 0.004 & 0.003 \\
\hline
\end{tabular}


-Arctic $=$ Antarctica
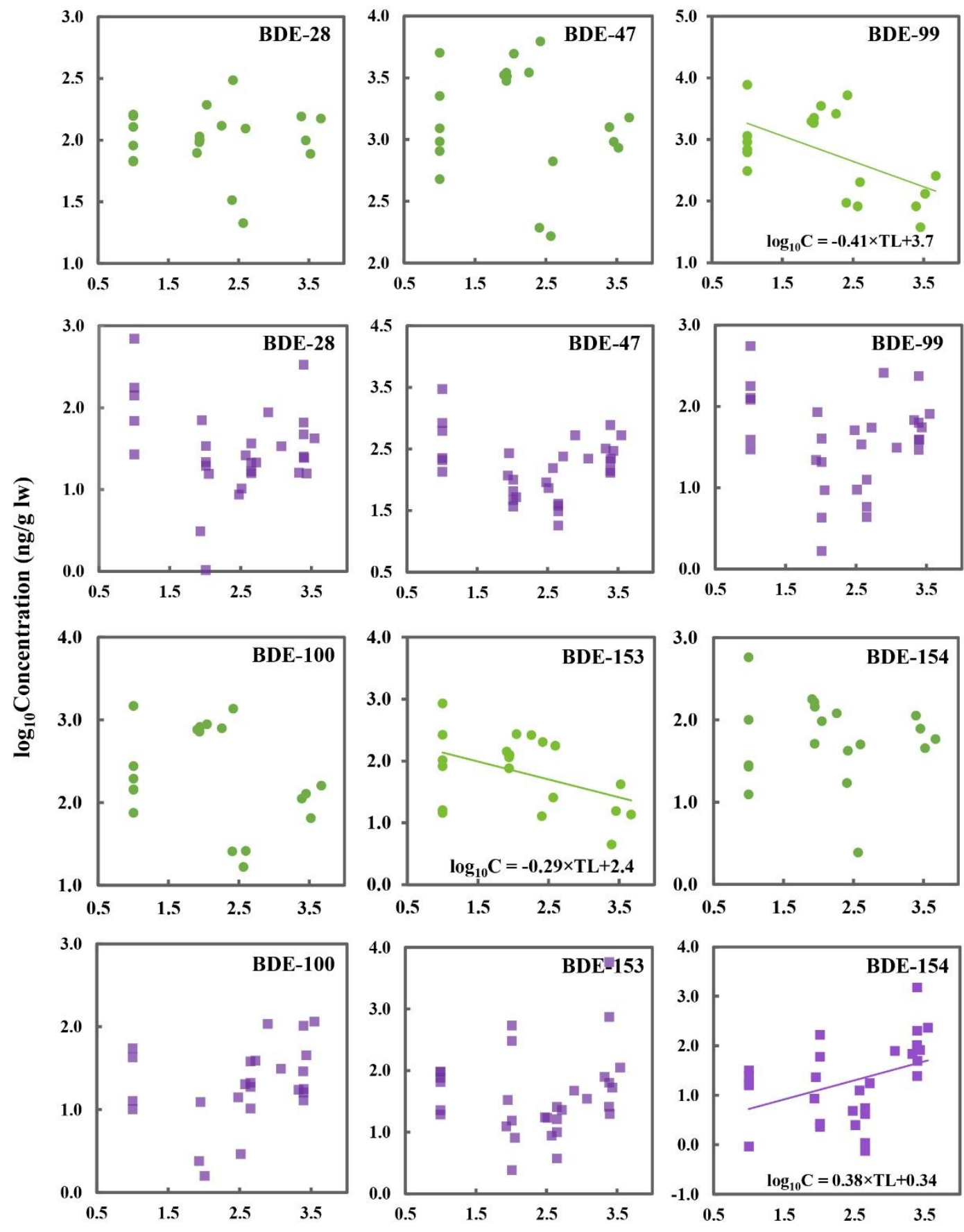

Trophic level

Figure S2(A). PBDE concentrations in marine organisms versus trophic levels in Polar Regions. 


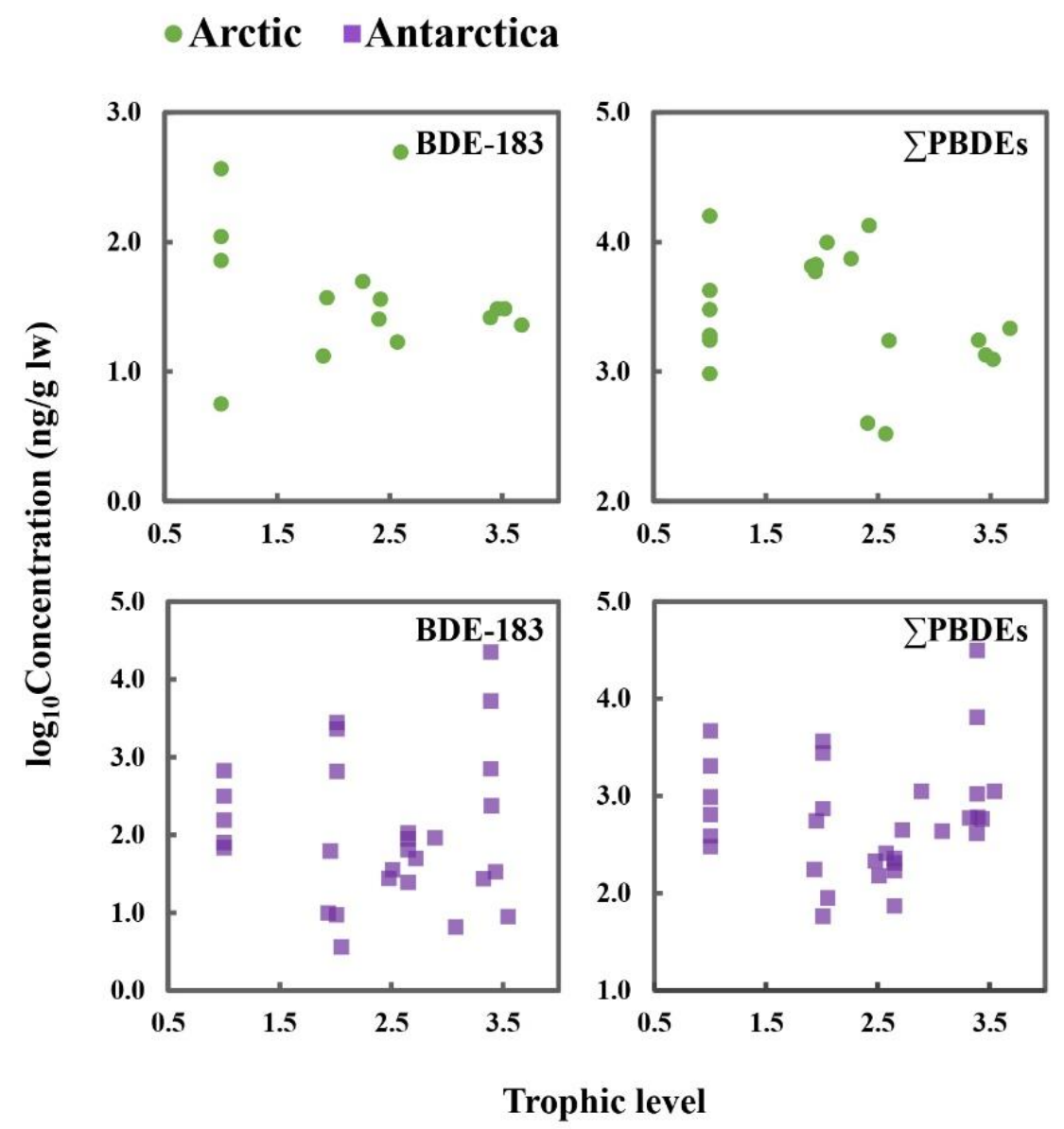

Figure S2(B). PBDE concentrations in marine organisms versus trophic levels in Polar Regions. 
-Arctic $=$ Antarctica
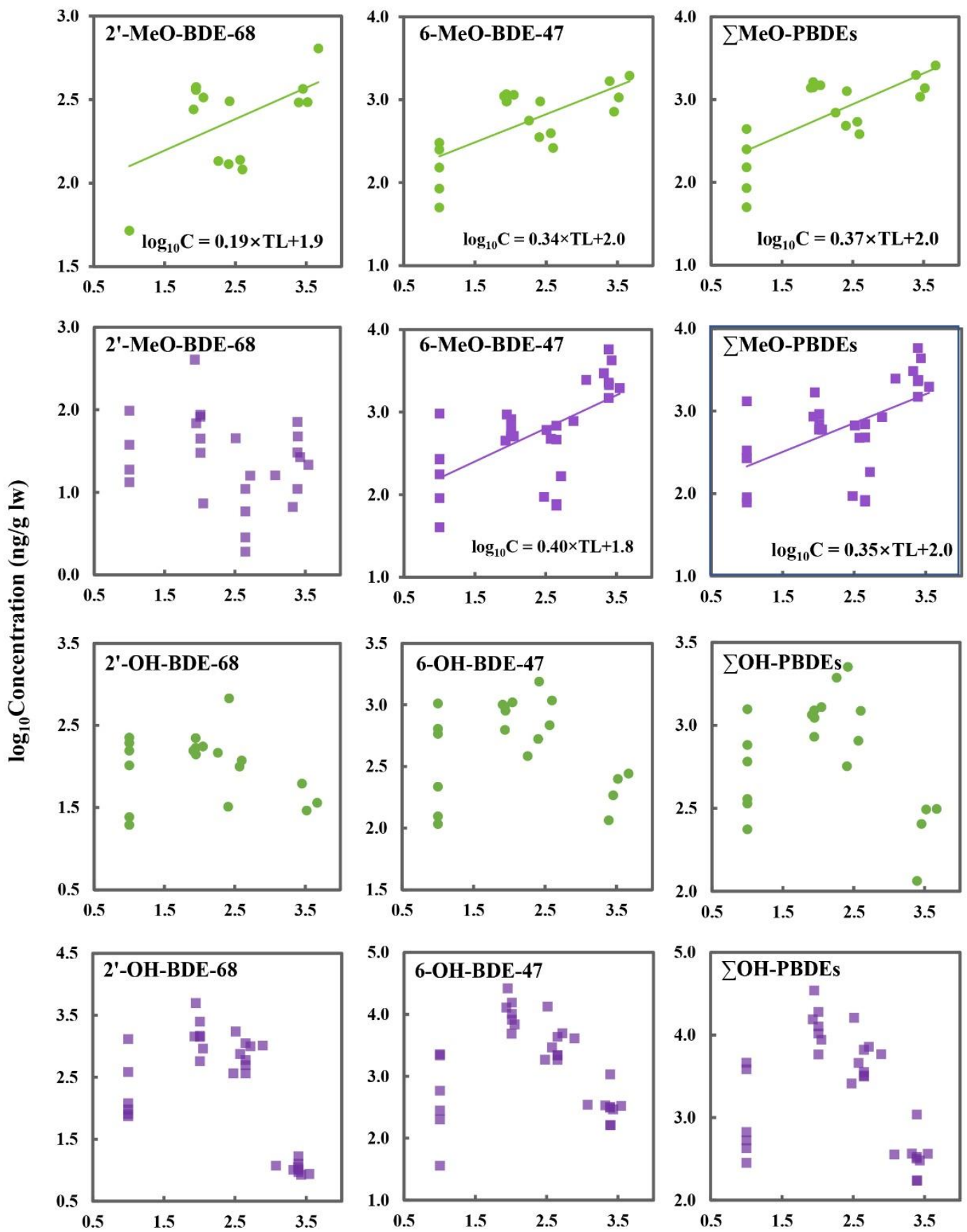

Trophic level

Figure S3. MeO-PBDE and OH-PBDE concentrations in marine organisms versus trophic levels in Polar Regions. 